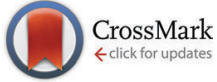

Cite this: Phys. Chem. Chem. Phys., $2014,16,19365$

\section{Hydrogen bond dynamics of superheated water and methanol by ultrafast IR-pump and EUV-photoelectron probe spectroscopy $\dagger$}

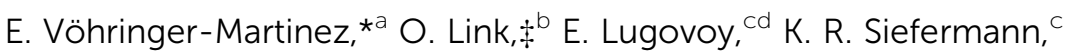 \\ F. Wiederschein, ${ }^{e}$ H. Grubmüller ${ }^{e}$ and B. Abel $^{\mathrm{cd}}$
}

\begin{abstract}
Supercritical water and methanol have recently drawn much attention in the field of green chemistry. It is crucial to an understanding of supercritical solvents to know their dynamics and to what extent hydrogen $(\mathrm{H})$ bonds persist in these fluids. Here, we show that with femtosecond infrared (IR) laser pulses water and methanol can be heated to temperatures near and above their critical temperature $T_{\mathrm{C}}$ and their molecular dynamics can be studied via ultrafast photoelectron spectroscopy at liquid jet interfaces with high harmonics radiation. As opposed to previous studies, the main focus here is the comparison between the hydrogen bonded systems of methanol and water and their interpretation by theory. Superheated water initially forms a dense hot phase with spectral features resembling those of monomers in gas phase water. On longer timescales, this phase was found to build hot aggregates, whose size increases as a function of time. In contrast, methanol heated to temperatures near $T_{\mathrm{c}}$ initially forms a broad distribution of aggregate sizes and some gas. These experimental features are also found and analyzed in extended molecular dynamics simulations. Additionally, the simulations enabled us to relate the origin of the different behavior of these two hydrogen-bonded liquids to the nature of the intermolecular potentials. The combined experimental and theoretical approach delivers new insights into both superheated phases and may contribute to understand their different chemical reactivities.
\end{abstract}

Received 12th May 2014, Accepted 17th July 2014

DOI: $10.1039 / c 4 c p 02063 d$

www.rsc.org/pccp chemistry and as reaction media for the decomposition of hazardous organic compounds and recovery of useful chemicals from industrial wastes. ${ }^{3}$ The study of supercritical water (SCW) is thus relevant to the widespread use of supercritical solvents in industrial reactions and separation science. SCW consists of highly excited molecules ${ }^{9}$ and is believed to be important in the geological formation of hydrocarbons. ${ }^{10-13}$ New forms of life have been found at deep-sea hydrothermal vents ${ }^{14-16}$ - the conditions at some of these locations are close to the critical point of water. ${ }^{14}$ Significant deuteration of simple organics with very little auxiliary reaction has been found in near-critical and supercritical $\mathrm{D}_{2} \mathrm{O} .{ }^{17}$

Since the unique properties of SCW and SCM as solvents ${ }^{4,5}$ in chemical reactions arise from fluctuations in the hydrogen bonding state and density (aggregate formation), information on their structures and dynamic properties is highly desired for understanding of the underlying mechanisms of chemical reactions and further development of supercritical fluids technology. ${ }^{6-8}$ In this context, it is crucial to know to what extent $\mathrm{H}$ bonds persist in these fluids. ${ }^{18}$ For water, this issue has already been addressed by neutron diffraction studies ${ }^{19,20}$ using the isotopic substitution technique. ${ }^{21,22}$ It has been realized that the interpretation of these data is in fact nontrivial. ${ }^{23}$ Beyond the problem of extracting $\mathrm{OO}, \mathrm{HH}$, and $\mathrm{OH}$ pair distribution functions from the raw data, it is unclear how 
to determine the extent of $\mathrm{H}$ bonds from the pair distribution functions. $^{24,25}$ X-ray diffraction data are also helpful, ${ }^{26-28}$ although X-rays do not efficiently detect the low- $Z$ hydrogen atoms. Thus, X-ray data provide somewhat indirect evidence of $\mathrm{H}$ bond formation. Another powerful technique for examining $\mathrm{H}$ bonds turned out to be vibrational spectroscopy - either infrared (IR) absorption ${ }^{29}$ or Raman scattering. ${ }^{30-32}$ The IR and Raman data have been analyzed to yield quantitative estimates of the extent of hydrogen bonding in supercritical water. ${ }^{28,30}$ However, there is no general agreement about the analysis of the various data sets. Molecular dynamics simulations and Monte Carlo calculations have provided further insight into hydrogen bonding. ${ }^{24,25,33-35}$ These methods generally find a reduced but non-negligible extent of hydrogen-bonding in supercritical water. ${ }^{18}$

Despite a large number of reports on SCW data at the molecular level, ${ }^{18}$ there are much fewer studies on SCM. Raman spectra, ${ }^{36}$ NMR chemical shift, ${ }^{37-39}$ relaxation ${ }^{40}$ measurements and MD simulations ${ }^{41,42}$ for SCM have shown that the number of hydrogen bonds of methanol decreases with increasing temperature, but that hydrogen bonds still remain even in the supercritical state. The microscopic structures of the clusters in SCM have been revealed in detail from neutron diffraction studies with isotopic substitution measurements. ${ }^{43}$ In the supercritical state at moderate densities, there are clusters of 3-5 molecules with half the average chain-length compared with that of liquid methanol at ambient temperature. Density fluctuations of SCM have also been reported by measurements of small-angle neutron scattering. ${ }^{44}$ Since most of the results on supercritical fluids are, however, obtained from static non time-resolved experiments yielding time- and ensemble averaged observables, time-resolved experimental studies on supercritical fluids such as methanol and water are desirable, as they allow resolving their dynamics on ultrafast timescales directly. In liquid water the ultrafast hydrogen bond dynamics after IR-excitation were studied successfully under ambient conditions ${ }^{45}$ and only recently in supercritical phases. ${ }^{46-48}$

As opposed to two previous studies by our group on water, ${ }^{47,48}$ which demonstrate that water can be heated with an IR laser significantly above the boiling point and even up to the critical point, the focus of the present work is the comparison between the ultrafast dynamics of the hydrogen bonded systems of methanol and water and the interpretation of the experimental findings with the help of molecular dynamics simulations. Finally, an attempt is made to use these findings to understand the different behaviors of hot water and methanol phases in chemical reactions in general. ${ }^{49}$

To study the dynamics of both superheated and near critical temperature phases, we performed time-resolved photoelectron spectroscopy on a liquid jet of water and methanol. The liquid jet is heated by the IR pump-pulse tuned to the absorption band of the $\mathrm{OH}$ stretch vibration of the liquids. The evolution of the hot phases is monitored by recording the photoelectron spectrum with a probe-pulse with a center wavelength of $32 \mathrm{~nm}$ (38.7 eV) obtained via high-harmonic generation (HHG). Due to the difficulty in measuring surfaces of volatile liquids under vacuum, photoelectron spectroscopy of the liquid phase is much less well established than for solid samples. ${ }^{51}$ Only since the development of the liquid micro-jet technique under vacuum by Faubel et $a .^{52}$ PES or ESCA experiments on liquids have emerged with growing success. ${ }^{50}$ Combining the liquid micro-jet technique ${ }^{53}$ and table-top high-order harmonic generation (HHG) of XUV radiation $^{54}$ in an ultrafast pump-probe spectroscopy experiment recently allowed us to add the dimension of time to the liquid interface PES technique. ${ }^{47,48,55,56}$

It has to be pointed out, that photoelectron spectroscopy is in general a surface sensitive technique due to the limited escape depth of photoelectrons. In water, the escape depth of photoelectrons with kinetic energies of about $20-30 \mathrm{eV}$ - the kinetic energies relevant to our experiment - is about a few nanometers ${ }^{57-60}$ and thus about several layers of water molecules from the surface only. ${ }^{61}$ Therefore, the experiment senses the interface and the condensed phase at $t=0$ and the evolution of the hot superheated phase at $t>0$. In ref. 48 we have discussed why and how this experiment is - in principle - able to monitor the liquid and the superheated fluid, as well as the evolving hot phase quantitatively via photoelectron spectroscopy.

\section{Experimental and theoretical methods}

The liquid micro-jet and the pump-probe beam geometry in front of the time of flight electron spectrometer under vacuum are displayed schematically in Fig. 1. A high-pressure pump (HPLC-pump) injects the liquid through a quartz nozzle at about 10 bar resulting in a micro-jet with a diameter of 15-20 $\mu \mathrm{m}$ moving at a velocity of $50 \mathrm{~m} \mathrm{~s}^{-1}$. Femtosecond laser pulses $\left(\lambda_{\mathrm{c}}=800 \mathrm{~nm}, 100 \mathrm{fs}\right)$ are split into pump and probe pulse. The IR pump wavelength is generated by a TOPAS and subsequent difference-frequency generation in a KTP crystal yields pulse energies between 15-40 $\mu \mathrm{J}$ at center wavelengths between $2.8-3.0 \mu \mathrm{m}$ and a pulse length on the order of $\sim 250 \mathrm{fs}$. The probe pulse is generated via high harmonic generation in argon. ${ }^{47}$ A EUV grating is used to select the 25th harmonic $(38.7 \mathrm{eV}, 32 \mathrm{~nm})$ and a toroidal mirror focuses it into the liquid jet (focus diameter $\sim 100 \mu \mathrm{m})$. IR-pump and EUV-probe pulse are overlapped in space and time on the micro jet in the vacuum chamber. The cross correlation between both pulses has a FWHM of about 500 fs. The EUV probe pulse ionizes the liquid sample and generates photoelectrons from the valence electrons of the liquid and some gas phase around the liquid micro-jet. As displayed in Fig. 1 they are detected by a time-of-flight (TOF) electron spectrometer after passing through a 100-150 $\mu \mathrm{m}$ skimmer at a distance close to the micro-beam $(500 \mu \mathrm{m}) .{ }^{47,48}$ Our time-of-flight (TOF) electron spectrometer allows to record the entire photoelectron spectrum (multiplex advantage) after IR-excitation. The TOF data are converted into binding energies by a calibration function. The liquid phase photoelectron signals of water are different from the respective gas phase signals, as they are significantly broader and shifted towards lower binding energies. ${ }^{51}$ The experimental setup and procedures are described in more detail in ref. 47 and 48. 

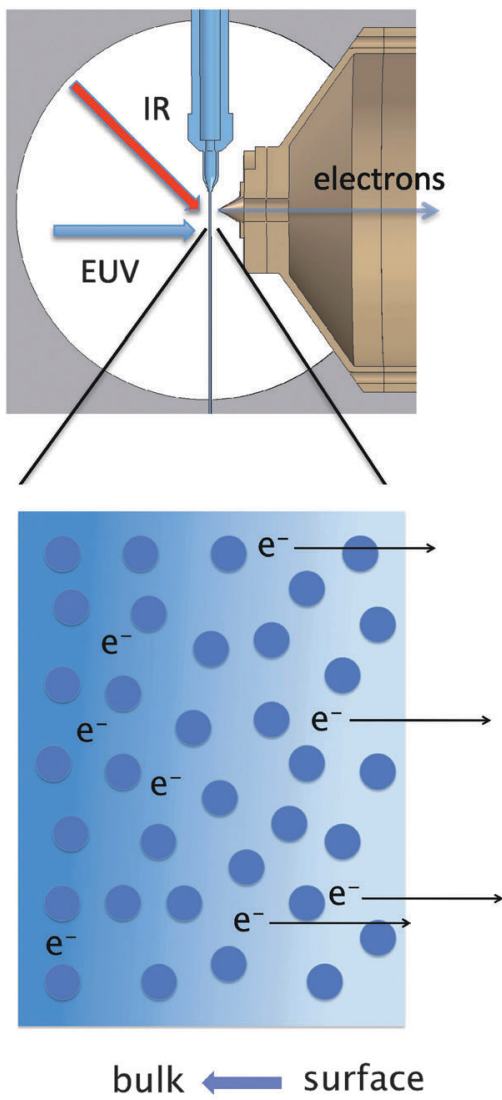

Fig. 1 Ultrafast ESCA near the liquid water interface under vacuum. The infrared pump and EUV probe (38.7 eV from high harmonics generation) overlap in time and space on the liquid water beam in front of the 100-150 $\mu \mathrm{m}$ skimmer (upper part of figure). The limited escape depth of the photoelectrons generated by the EUV probe pulse is shown schematically (lower part). The micro skimmer is in close proximity to the water beam $(\approx 500 \mu \mathrm{m})$. For details on the experiment and experimental setup see the text.

Molecular dynamics (MD) simulations were performed with the GROMACS simulation package. ${ }^{62}$ A rectangular box of the corresponding liquid with the dimensions of $30 \times 4 \times 4 \mathrm{~nm}$ was first equilibrated at $300 \mathrm{~K}$ and 1 bar with the Berendsen coupling schemes ${ }^{63}$ and then heated up under constant volume to the temperatures of $775 \mathrm{~K}$ for water and $650 \mathrm{~K}$ for methanol employing the interaction potentials given in the respective SPC- and the OPLS-model. ${ }^{64,65}$ Simulations performed with a polarizable model based on classical Drude oscillator, ${ }^{66}$ revealed very similar dynamics. The equilibrated simulation boxes of water $(775 \mathrm{~K})$ and methanol $(650 \mathrm{~K})$ were elongated in $x$-direction to $500 \mathrm{~nm}$ and the liquids were placed in the middle of the box, allowing for a phase evolution along both sides of the $x$-axis (periodic boundary conditions in the $y, z$ dimension). These elongated boxes were simulated in an NVE-ensemble for 100 ps. A simulation with a larger water box $(30 \times 12 \times 12 \mathrm{~nm}$, approx. 500000 atoms) did not show any difference in the dynamics of the phase evolution, such that finite size effects are not expected to play a significant role. Therefore, thirteen simulations of the smaller box of each liquid were performed, resulting in 26 independent phase evolutions of 100 ps each.
This number of simulations yielded converged analysis results (for details see ESI $\dagger$ ).

\section{Results and discussion}

Tuning the intensity and wavelength of the IR-pump pulse allows varying the amount of energy deposited in the liquid jet. In Fig. 2a the liquid jet in front of the micro skimmer is displayed. The pulse profile in the laser focus has been measured precisely with a knife-edge technique. ${ }^{8}$ The fraction of the pulse energy hitting the liquid jet was determined from the jet diameter $(\sim 15-20 \mu \mathrm{m})$, the focus diameter and the intensity distribution of the IR-laser pulse (Fig. 2a). It is on the order of $25 \%$ of the pulse energy. The absorption profile of the IR-light in the liquid jet was estimated from literature values for the absorption coefficients of water and methanol at the respective wavelength. It was found that the deposited energy is constant over the first $10 \mathrm{~nm}$, and thus over the distance probed by the EUV probe pulse. From the deposited energy per volume of the liquid and the heat capacities we estimated the temperatures of the heated liquids. ${ }^{67}$ What has to be kept in mind is that temperatures of the water and methanol jets prior to heating by the IR-pump pulse are different and depend upon the liquid, the distance from the nozzle exit and the diameter of the jet (Fig. $2 b$ - values are calculated according to ref. 53 and 56). Typically, pump and a)

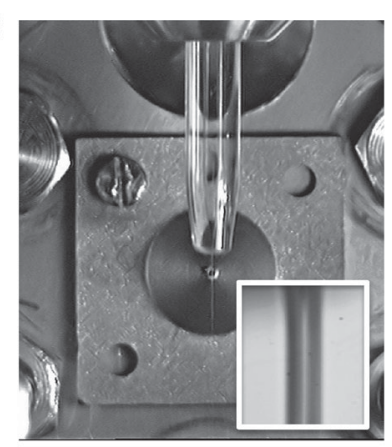

b)

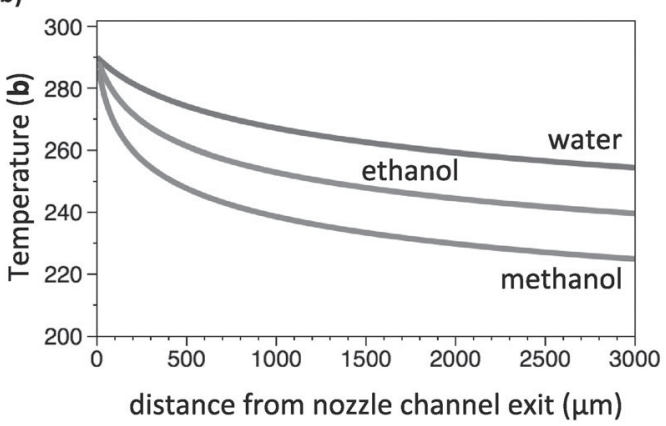

Fig. 2 (a) Left: liquid water nozzle and micro jet in front of the electron spectrometer. The inset shows the water jet (contraction) at the nozzle exit. The liquid jet's diameter is about $14 \mu \mathrm{m}$. Right: IR-laser intensity distribution projected (hitting) the water beam. The geometry is important for estimating the internal energy content of the illuminated liquid micro jet. (b) Liquid jet temperatures as a function of the distance from the nozzle exit. The temperatures have been calculated with an evaporation model describing evaporative cooling of a cylindrical liquid beam. ${ }^{53,56}$ 
probe pulse overlap on the jet at a distance of 1-2 $\mathrm{mm}$ from the nozzle exit.

For the experimental data shown in Fig. 3 (water) the wavelength of the IR-pulse was $2830 \mathrm{~nm}$ and the deposited energy was estimated to be on the order of $\sim 36 \mathrm{~kJ} \mathrm{~mol}^{-1}$. This results in a temperature of the heated liquid of $740 \pm 100 \mathrm{~K}$, which is near or even above the critical temperature of water of $T_{\mathrm{c}}=647 \mathrm{~K}$. For methanol, a wavelength of $2950 \mathrm{~nm}$ was chosen and the deposited energy was estimated at $\sim 26 \mathrm{~kJ} \mathrm{~mol}^{-1}$ corresponding to a temperature of $550 \pm 100 \mathrm{~K}$, which is near the critical temperature of $T_{\mathrm{c}}=512 \mathrm{~K}$. Achieving higher temperatures in methanol was not possible in our experiment due to limitations in the available pulse energies at wavelengths of $\sim 3000 \mathrm{~nm}$ and the absorption coefficient of $\mathrm{MeOH}$ at this wavelength, which is low compared to water. The large error bars are mainly attributed to uncertainties in the initial temperatures of the liquid jet and uncertainties in the estimation of the deposited energy.

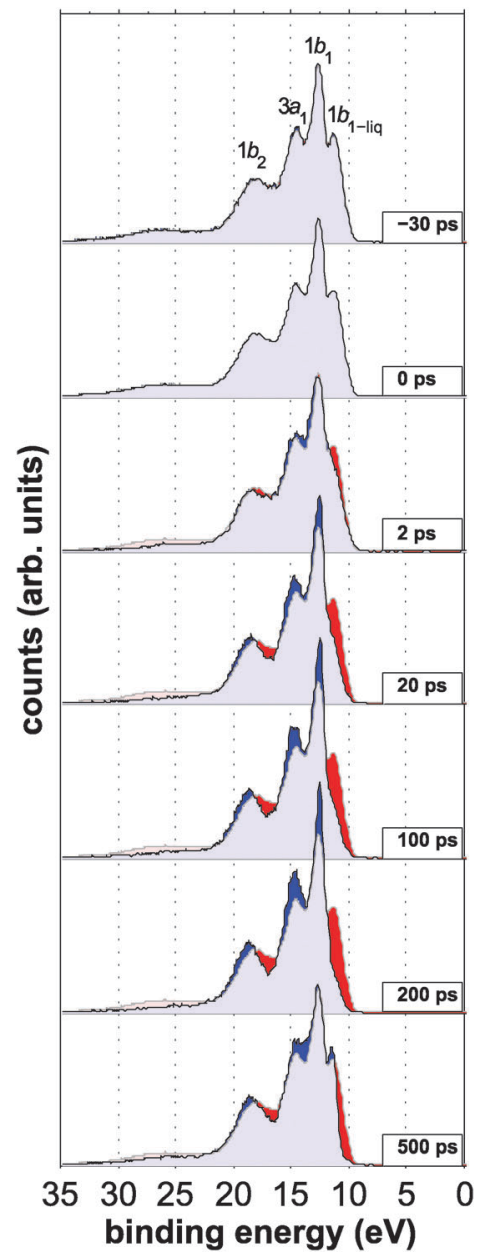

Fig. 3 Time-resolved photoelectron spectra revealing expansion dynamics of superheated water $\left(\lambda_{\mathrm{IR}}(\mathrm{pump})=2830 \mathrm{~nm}\right.$, deposited energy corresponding to $740 \pm 100 \mathrm{~K})$. The time between IR pump and EUV probe pulse increases from top to bottom. The changes relative to the reference spectrum at negative delay are indicated in red (decrease in intensity) or blue (increase in intensity). $1 b_{1}, 3 a_{1}, 1 b_{2}$, and $1 b_{1-\text { liq }}$ denotes the valence states of water in the gas phase and in the liquid phase $\left(1 \mathrm{~b}_{1-\text {-iq }}\right)$.
We note that the present extreme matter conditions cannot be obtained in static high-pressure cells containing supercritical gas/fluids. ${ }^{7,68}$ Time-series of photoelectron spectra for the excitation of water are displayed in Fig. 3. The spectrum at the top is a reference spectrum (un-heated) recorded at negative time delays. The spectrum consists of a superposition of photoemission lines from the liquid and the gas phase (which is present around the liquid jet). The ground state electron configuration of water is: $\left(1 a_{1}\right)^{2}\left(2 a_{1}\right)^{2}\left(1 b_{2}\right)^{2}\left(3 a_{1}\right)^{2}\left(1 b_{1}\right)^{2}$. In Fig. 3, photoelectron signal originates from the HOMO-2 $\left(1 b_{2}\right)$, HOMO-1 $\left(3 a_{1}\right)$ and HOMO $\left(1 b_{1}\right)$ of the water molecule. In the gas phase, the vertical binding energies are $18.55 \mathrm{eV}$ $\left(1 b_{2}\right), 14.73 \mathrm{eV}\left({ }^{3} \mathrm{a}_{1}\right)$ and $12.61 \mathrm{eV}\left(1 \mathrm{~b}_{1}\right) .{ }^{79}$ The corresponding liquid signals are located at lower binding energies: $17.34 \mathrm{eV}$ $\left(1 \mathrm{~b}_{2 \text {-liq }}\right), 13.50 \mathrm{eV}\left(3 \mathrm{a}_{1 \text {-liq }}\right)$ and $11.16 \mathrm{eV}\left(1 \mathrm{~b}_{1 \text {-liq }}\right) .{ }^{51}$ The $1 \mathrm{~b}_{1 \text {-liq }}$ is thus somewhat separated from the other signals and can be nicely followed in the pump-probe experiment. ${ }^{69}$ In order to facilitate this, the time-resolved spectra have been plotted/color coded in a way that red displays a decrease and blue an increase of intensity, with respect to the reference spectrum at negative delays. Within the first 100 ps the intensity of the $1 \mathrm{~b}_{1 \text {-liq }}$ peak decreases, while the intensity of the corresponding $1 b_{1}$ gas phase signal increases. A similar trend is observed for the other liquid and respective gas phase signals, although not as clearly since the signals strongly overlap. We note that the spectra detected at a time-delay of about 100 ps resemble the spectra that we recorded of pure gas phase water, except for a small shoulder on the low-binding energy side of the $1 b_{1}$ gas signal. At time delays $>300 \mathrm{ps}$, a new signal in the binding energy range between $1 b_{1}$ gas and $1 b_{1-\text { liq }}$ appears and increases in intensity as a function of pump-probe delay. As can be seen in the spectrum at $500 \mathrm{ps}$, this signal is narrower than the initial $1 b_{1-l i q}$ signal and located at slightly higher binding energies. The signal shape and position is consistent with the $1 b_{1}$ signal detected for water clusters. ${ }^{70-72}$ Furthermore, the binding energy position of the new cluster feature systematically shifts from higher to lower binding energies as a function of pumpprobe delay, indicating an increasing cluster size.

Our interpretation of the experimental data is the following: The deposition of energy into the liquid jet is ultrafast, as it happens within the 250 fs pulse length of the IR pump-laser. Intramolecular vibrational energy redistribution from the excited $\mathrm{OH}$-stretch to all degrees of freedom of the water is ultrafast. ${ }^{73,74}$ Within this short time of energy deposition, the system cannot significantly expand. Assuming that the volume stays constant over the time of energy deposition, the system is transferred into a near critical or supercritical state. Note that the term "near critical or supercritical" refers to the temperature only since it is unlikely that this state corresponds to an equilibrium state with $\mathrm{T}, \mathrm{p}, \mathrm{V}$, being all at or above their critical values within the timespan of the excitation pulse (the hot volume expands somewhat on very short timescales). Even though the thermodynamic definition of the critical point cannot be applied here, we call the superheated phase the near critical temperature phase for distinction. Simultaneously, expansion of the hot liquid sets in and continues over the entire series of spectra. As a consequence, 
the intensity of the $1 \mathrm{~b}_{1 \text {-liq }}$ signals decreases. The rapid expansion leads to a cooling of the hot phase. At a certain time ( 100-300 ps) the temperature falls significantly below the critical temperature and a phase separation in the inner part of the expanding cloud occurs, accompanied by the appearance of the cluster signal in the photoelectron spectra.

In order to determine timescales for the decay of the liquid, the intensity at representative binding energies is plotted as a function of pump-probe time delay in Fig. 5. Because of the spectral overlap of most lines we chose a binding energy $(10.5 \mathrm{eV})$ at the low-energy wing of the (static) liquid $1 \mathrm{~b}_{1 \text {-liq. }}$ photoemission line and the $1 b_{1}$ gas phase emission maximum (12.6 eV). The chemical shift at $10.5 \mathrm{eV}$ is predominantly from liquid water molecules and monitoring this spectral region is a good measure of the evolution of a superheated liquid phase. As is obvious from Fig. 5 the decay at $10.5 \mathrm{eV}$ does not follow a simple exponential decay. The polydispersity of the evolving system is better described by a stretched exponential. Fitting the decay at $10.5 \mathrm{eV}$ with a stretched exponential $y=\exp \left(-1 / \tau \cdot t^{b}\right)$ leads to characteristic parameters of $\tau=1.45$ ps and $b=0.15$.

A stretched exponential with $b=1$ (with $b$ being a polydispersity parameter) is a single exponential decay. The smaller $b$, the larger is the polydispersity of the heterogeneous lifetime distribution corresponding to a specific "average" lifetime $\tau$. The low $b$ parameter thus suggests that the system is decaying with a large distribution of time constants. This finding is consistent with density fluctuations within the expanding hot phase, which proceed on multiple different timescales. The comparison of the data with the molecular dynamics simulations described below appears to favor a polydisperse distribution of species (water in various mesoscopic and nanoscopic forms) decaying with a distribution of time constants. We have no evidence that the kinetics may be governed by other mechanistic reasons such as reported for phase transitions in ice by the group of Laubereau. ${ }^{75}$

Fig. 4 shows time-resolved photoelectron spectra of methanol heated to a temperature of about $550 \pm 100 \mathrm{~K}$. Similar to water, the photoelectron spectra of methanol also consist of a superposition of the liquid and the gas phase photoelectron emission lines. The assignment of the photoemission lines $\left(5 a^{\prime}, 1 a^{\prime \prime}, 6 a^{\prime}\right.$, $7 \mathrm{a}^{\prime}$, and $2 \mathrm{a}^{\prime \prime}$ ) has been taken from ref. 76. Beyond the $2 \mathrm{a}^{\prime \prime}$ gas phase photoelectron emission line towards lower binding energies a liquid band $2 a^{\prime \prime}$ liq is observed. Following the evolution of this signal, one observes a markedly slower and less pronounced change compared to water. The spectra indicate that the hot liquid methanol phase directly disaggregates into a broad distribution of clusters and some gas within about 200300 ps. For longer time delays only a small change in the signal is observed. Fig. 6 shows the intensity at $9.5 \mathrm{eV}$ representing the liquid and $10.95 \mathrm{eV}$ (gas phase) as a function of pump-probe time delay. The dynamical evolution of the methanol spectra appears to be slower compared to water. This changed timescale of the decay manifests itself in the parameters for the stretched exponential fit, namely $\tau=10$ ps and $b=0.3$ for methanol.

Taken together, superheated and near critical temperature water initially forms a dense hot phase with spectral features resembling mostly those of gas phase water. On longer timescales,

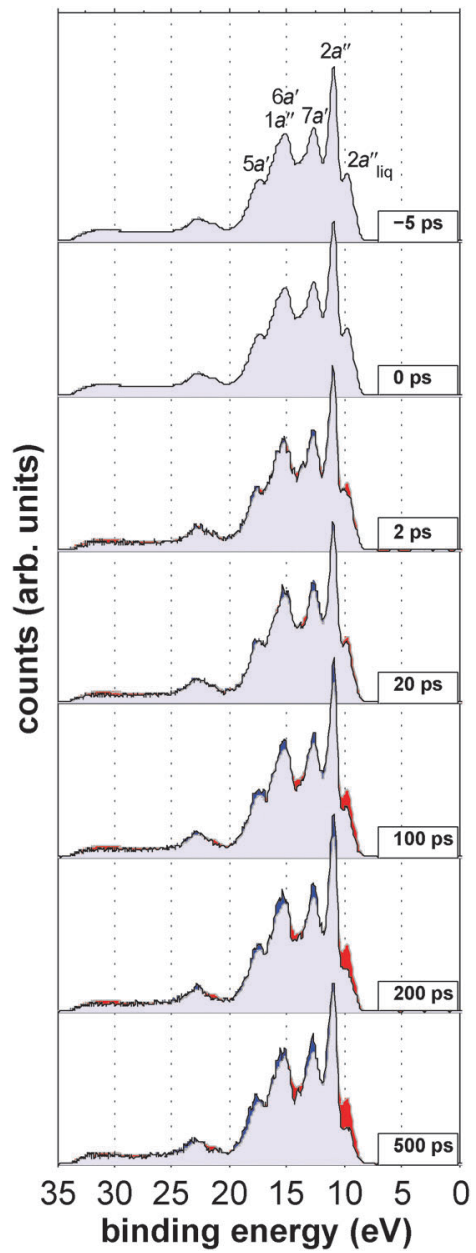

Fig. 4 Time-resolved photoelectron spectra revealing expansion dynamics of methanol heated to temperatures near $T_{\mathrm{C}}\left(\lambda_{\mathrm{I}}(\mathrm{pump})=2950 \mathrm{~nm}\right.$, deposited energy corresponding to $550 \pm 110 \mathrm{~K}$ ). The time between IR pump and EUV probe pulse increases from top to bottom. The changes relative to the reference spectrum at negative delay are indicated in red (decrease in intensity) or blue (increase in intensity). For assignments see the top traces of each panel.

this phase was found to condense into clusters, whereas the cluster size increases as a function of time. In contrast, superheated and near critical temperature methanol initially forms a broad distribution of cluster sizes and some gas. This finding is surprising and somewhat counterintuitive because hydrogen bonding in methanol is much less pronounced than in water. Therefore, we had expected that the dynamics of the dense phase evolution would be significantly faster resulting in large amounts of primary monomers due to the lower density of hydrogen bond interactions in liquid methanol. Our conclusion from the present findings is that intrinsic (structural and dynamical) features of superheated phases, which may be relevant and characteristic for a number of situations in which reactions take place in supercritical environments, manifest themselves in the timescales of our experiments.

To shed light on these surprising and unexpected experimental observations, molecular dynamics (MD) simulations were performed (for details see the Theoretical Methods section). 


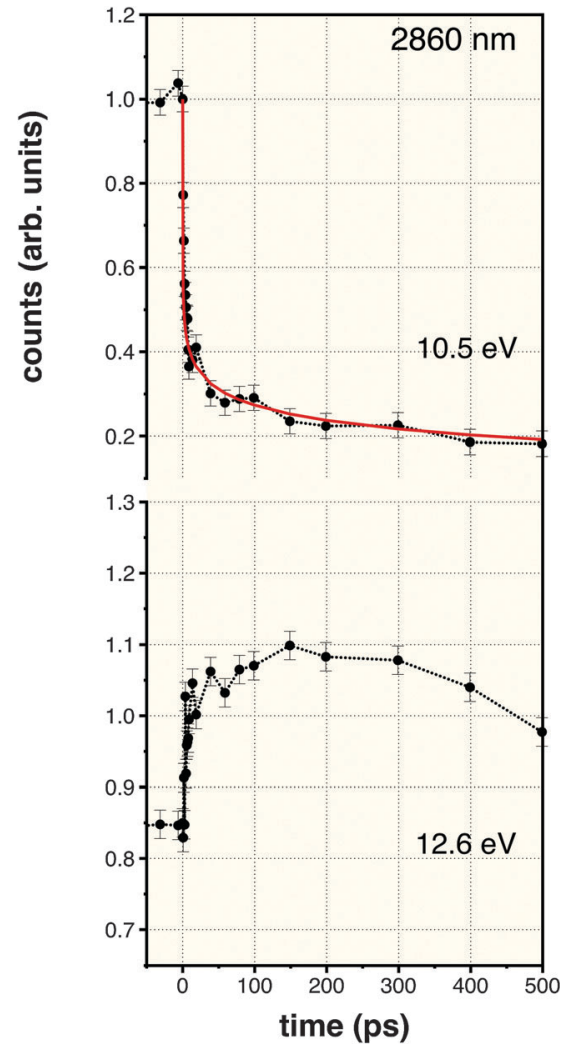

Fig. 5 Water: Photoelectron intensities at specific binding energies as a function of pump-probe delay. The intensities were extracted from the dataset displayed in Fig. 3. Top: Intensity at $10.5 \mathrm{eV}$ is representative of the liquid phase. The red line is a stretched exponential fit. Bottom: Intensity at $12.6 \mathrm{eV}$ is representative of the gas phase. Error bars indicate uncertainties of the experiment.

Previous studies have already shown the ability of employed SPC (water) and OPLS (methanol) models to describe correctly the structure and thermodynamics of these liquids under supercritical conditions. ${ }^{38,42,77}$ In order to compare the dynamics in water and methanol, simulations were performed at temperatures of $T_{\mathrm{H}_{2} \mathrm{O}}=775 \mathrm{~K}$ and $T_{\mathrm{MeOH}}=650 \mathrm{~K}$. For both liquids, these temperatures are about $120 \mathrm{~K}$ and $140 \mathrm{~K}$ higher than their critical temperatures, respectively. To match the fast experimental laser heating of the liquids a rectangular box of each liquid at normal densities were heated to the respective temperatures $\left(T_{\mathrm{H}_{2} \mathrm{O}}=775 \mathrm{~K} ; T_{\mathrm{MeOH}}=650 \mathrm{~K}\right)$, neglecting molecules possibly present in the vapor phase during the heating in the experiment (due to the dimensions of the simulation box only a few of them would be present at the liquid-vapor interface). Since the methanol temperature in the experiment was $T=550 \mathrm{~K} \pm 100 \mathrm{~K}$, we also performed methanol simulations at $500 \mathrm{~K}$ (for details on the analysis results see the ESI $\dagger$ ). Afterwards the expansion of these superheated liquids was investigated as described in the Theoretical Methods section.

The obtained simulations were then analyzed with respect to the hydrogen bond dynamics and the formation of aggregates, since local density of water molecules and hydrogen bonding are responsible for the measured shifts in the binding energies

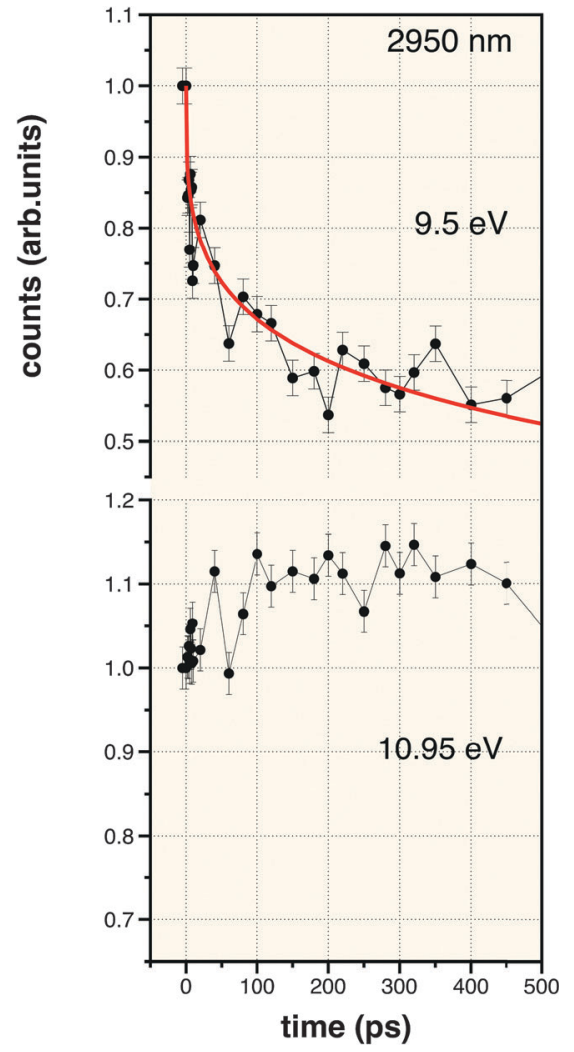

Fig. 6 Methanol: Photoelectron intensities at specific binding energies as a function of pump-probe delay. The intensities were extracted from the dataset displayed in Fig. 4. Top: Intensity at $9.5 \mathrm{eV}$ is representative of the liquid phase. The red line is a stretched exponential fit. Bottom: Intensity at $10.95 \mathrm{eV}$ is representative of the gas phase. Error bars indicate uncertainties of the experiment.

of the electrons. As outlined above, the molecules that contribute to the measured photoelectron spectra and therefore the experimental observable belong only to the first mono layers of the liquid phase (the escape length of the electrons is $\sim 1 \mathrm{~nm}) .{ }^{58}$ Since the escape length is only known under normal conditions, in the following we will assume that this escape length depends only on the number of molecules (projected back at each time step onto the surface at $t=0$ ) and not on their temperature neither their density. We take the number of molecules in the first nanometer of the liquid phase under normal conditions as a reference and count in the simulations of the phase evolution into vacuum for each picosecond the first molecules from the vacuum towards the condensed phase until the reference number is reached. These molecules are used for the subsequent picosecond resolved analysis described below. The results are not expected to be significantly different if more molecules/mono layers, e.g., within the first two nanometers are considered (due to the uncertainties of the escape depth of electrons in photoelectron emission spectra).

Fig. 7 shows the hydrogen bond dynamics (A) and the formation of aggregates (B) for the superheated and near critical temperature water simulations at $775 \mathrm{~K}$ (left) and 


\section{Water:}

A

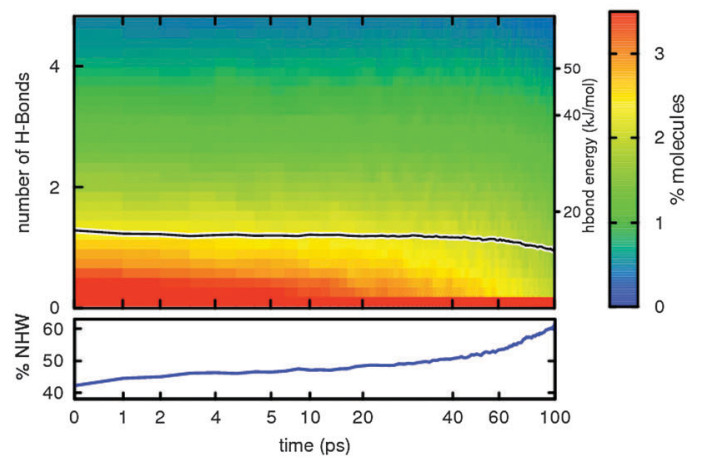

B

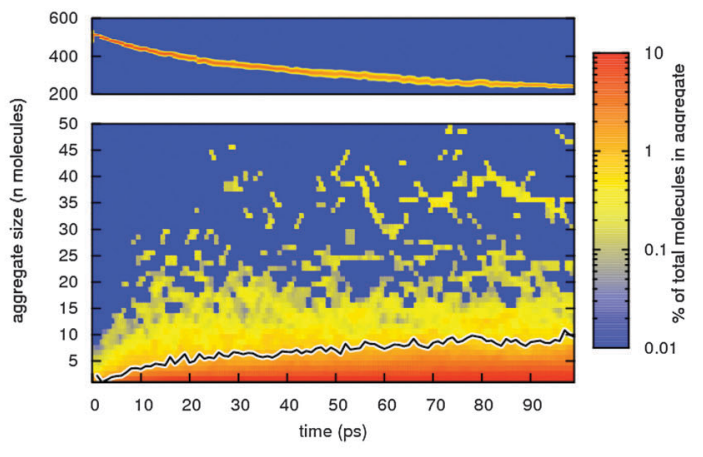

\section{Methanol:}

A

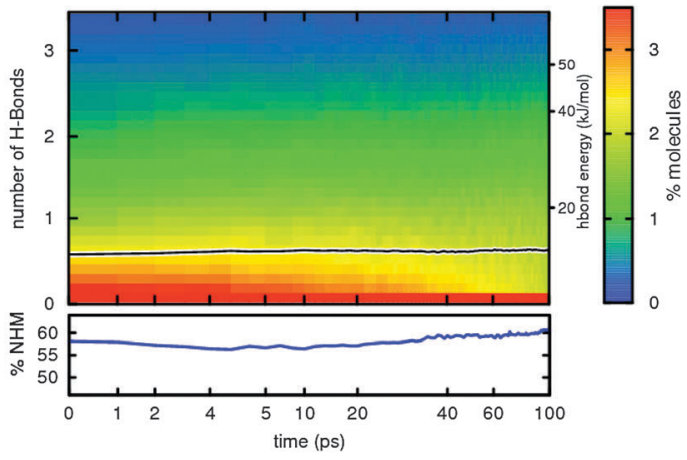

B

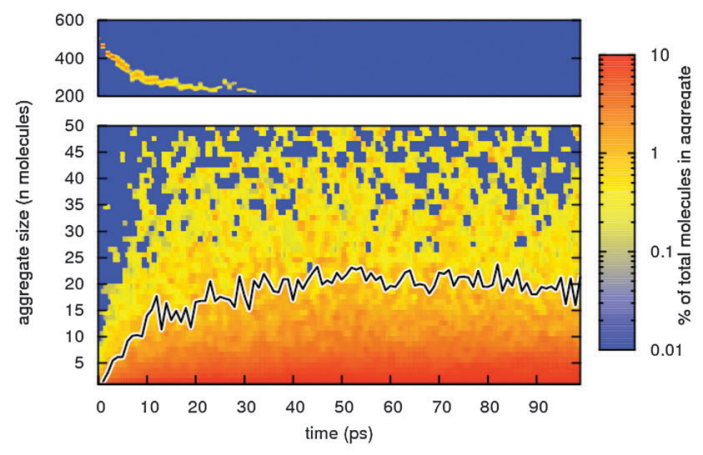

Fig. 7 (A) Top: distribution of molecules with a certain number of hydrogen bonds in the water simulations at $775 \mathrm{~K}$ and for methanol at $650 \mathrm{~K}$ (color coded). The number of hydrogen bonds is calculated from the energy of a hydrogen bond estimated by the Espinosa equation (right scale on plot) divided by the average energy of a hydrogen bond at $300 \mathrm{~K}$ and 1 bar. The black line shows the average of the distribution. Bottom: percentage of water (NHW) or methanol (NHM) molecules, which are not hydrogen bonded. (B) Top: aggregates larger than 200 molecules representing the condensed phase fitted with a Gaussian function at each time step. Bottom: distribution of small aggregate sizes in the water and methanol simulations. The ratio of molecules, which belong to an aggregate of a certain size is color coded up to aggregate sizes of 50 molecules and the average aggregate size up to this size is shown as a black line.

methanol at $650 \mathrm{~K}$ (right). Fig. 7A displays for each liquid the distribution of molecules with a certain number of hydrogen bonds color-coded along the simulation time. Hydrogen bonds were identified by a geometric criterion (OO-distance $<3.5 \AA$ and angle between donor-hydrogen-acceptor $<30^{\circ}$ ). To distinguish also between the strength of the hydrogen bonds with respect to each other, an energy function dependent on the hydrogen-acceptor distance was used (Espinosa equation ${ }^{78}$ ). The number of comparable hydrogen bonds per molecule displayed in Fig. 7A on the left ordinate is calculated from the energy of the hydrogen bonds in the molecule (shown on the right ordinate) divided by the average energy of a hydrogen bond of the corresponding liquid at $300 \mathrm{~K}$ and 1 bar. At the bottom of Fig. 7A the fraction of non-hydrogen bonded water (NHW) and methanol molecules (NHM) is shown. Comparison of the hydrogen bond dynamics of the two liquids reveals that the number of hydrogen bonds decreases in superheated water during the simulation and the fraction of isolated molecules (NHW) strongly increases. After 100 ps only $40 \%$ of the molecules are involved in hydrogen bonds, and will therefore contribute to the $1 \mathrm{~b}_{1 \text {-liq }}$ peak in the measured photoelectron spectrum. In methanol, however, the overall number of hydrogen bonds hardly changes during 100 ps.
At the bottom of Fig. 7 the aggregate size distribution for water (left) and methanol (right) during the simulations is shown. A (high temperature) "aggregate" consist of molecules with distances to the nearest neighbors smaller than $3.5 \AA$ A. For clarity, the aggregate size distribution is split into aggregates larger than 200 molecules in size at the top that represent the superheated condensed phase (fitted with a Gaussian function) and smaller aggregates $(n<50)$ together with their average value (bold black line). The time-dependence of the aggregate size distributions for the two hydrogen-bonded liquids is clearly different. Superheated water displays a smooth, moderate decrease in the size of the large aggregates and a slow increase in the number of smaller aggregates. The average size of the smaller aggregates after $100 \mathrm{ps}$ is about 10 molecules. The large aggregates in methanol, in contrast, disappear almost completely after the first $30 \mathrm{ps}$ and a pronounced increase in the number of small aggregates with an average aggregate size of 20 molecules after $100 \mathrm{ps}$ is observed. This result has to be discussed in consideration of the hydrogen-bond dynamics. The fast formation of small aggregates in methanol is accompanied by the preservation of the hydrogen bonds of the molecules forming the aggregate. Water, however, builds mostly monomers and aggregates appear only on longer time-scales, 
A

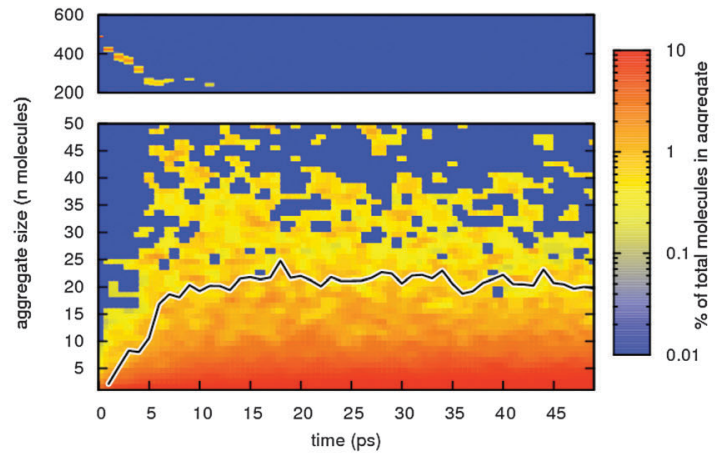

C

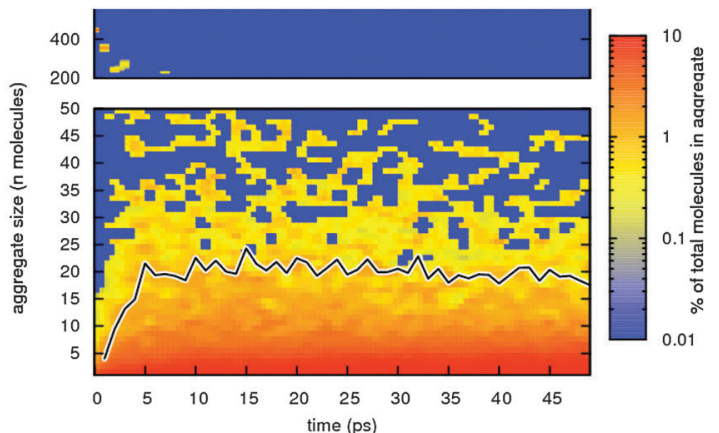

B

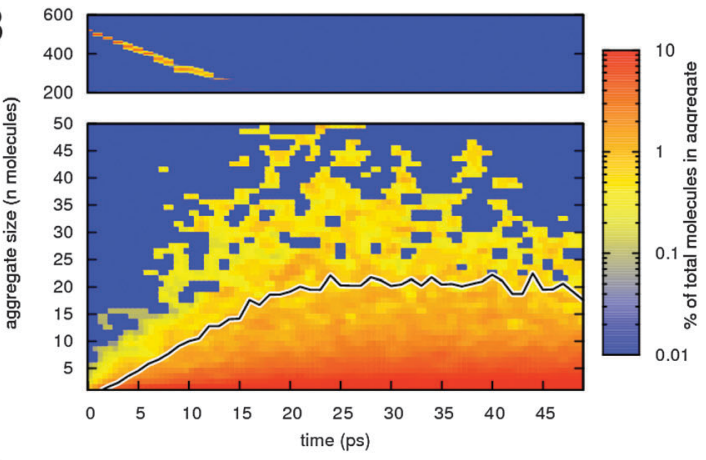

D

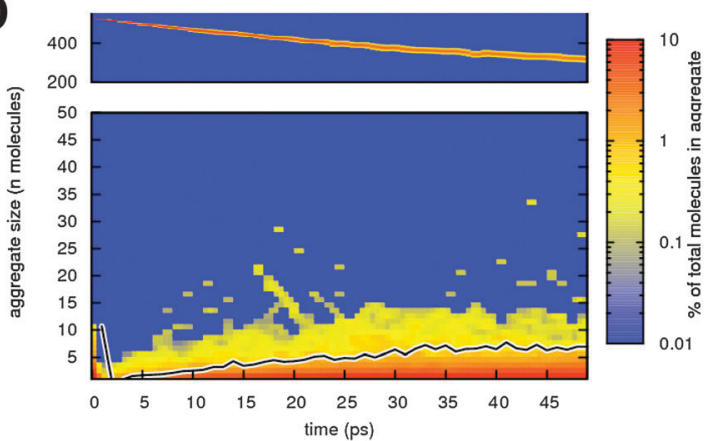

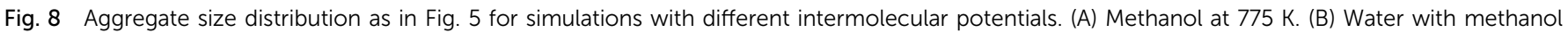

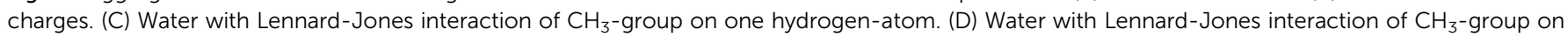
both hydrogen-atoms.

which were also observed in the experiment. This behavior is in perfect agreement with the low parameters $b$ of the stretched exponentials obtained in Fig. 5 and 6 . The small value of $b$ obtained for water in the experiment suggests larger polydispersity that originates from the variety of aggregate sizes observed in the water simulations as a function of time.

In a second step we also addressed the origin of the different behavior between the two liquids. Comparison of their intermolecular potentials shows that if the $\mathrm{CH}_{3}$-group in methanol is treated as one interaction site, as is done in the employed OPLS-model, the two simulated liquids only differ in the parameters of the intermolecular potentials and only slightly in the molecular geometry. This suggests that the dynamics are mainly driven by the different intermolecular interactions. To analyze which property of the potential is responsible for the observed behavior, the parameters of the SPC-water model were changed systematically towards the methanol parameters in simulations at $775 \mathrm{~K}$ (as a "reference" Fig. 8A displays the result of methanol at this temperature). First the atomic charges of water were replaced with the charges of the methanol molecule resulting in a more "polar" and a "hydrophobic" hydrogen atom. These simulations displayed the same fast increase in the number of small aggregates as in methanol (see Fig. 8B). When the charges of water were conserved but the LennardJones interactions of one hydrogen atom were modified to match the ones of the $\mathrm{CH}_{3}$-group the same results were obtained (Fig. 8C). In the last simulation the Lennard-Jones interactions were applied to both hydrogen atoms (Fig. 8D). Here, the same slow decrease in the size of the large aggregates as for water was observed. This suggests that the formation of aggregates depends on the symmetry of the intermolecular potential. If two equally strong interactions at the hydrogen atoms are present the phase evolution is characterized by the formation of monomers and a slow decrease in the size of the remaining "liquid" phase. This is accompanied with an increase of the entropy and therefore a reduction of the free energy of the system. If the interactions at the hydrogen atoms differ either by varying the electrostatic or the Lennard-Jones interactions, the phase evolves through a fast formation of large aggregates, where the stronger interaction is preserved.

If methanol forms aggregates on a very short timescale, the question remains why these can hardly be distinguished from the initial liquid phase in the experimental spectra. The dynamics of the hydrogen bonds displayed in Fig. 7A shows that the fast formation of aggregates preserves the hydrogen bonds of the initial superheated and near critical temperature fluid phase. Given that the shift in the binding energy of the electrons is mainly due to hydrogen bonds the newly formed methanol aggregates would display the similar spectral signature as the liquid phase, and could therefore not be distinguished from the initial phase.

\section{Summary and conclusions}

The goal of the present work was to shed light on the dynamics of superheated phases of hydrogen bonded liquids and to help find and understand the hydrogen bond dynamics that should 
also be relevant for chemical reactions in these fluids. Here, we investigated the timescale of evolution of superheated water and methanol phases, their evolving hydrogen bonding network, as well as their energetics providing insights about their initial "extreme state". We are aware of the fact that an ultrafast (non-equilibrium) evolving laser-heated and extremely hot phase of a hydrogen bonded liquid such as water and methanol cannot easily be compared with a true supercritical phase of water and methanol (in thermodynamical equilibrium). Clearly, the conditions in the laser experiment are not in thermal/thermodynamic equilibrium and moreover not even exactly supercritical, because while the deposited internal energy corresponds to supercritical temperatures, the remaining variables' volume and pressure are certainly not those at or close to the critical point. We therefore repeatedly and very clearly referred to a superheated and near critical phase of water and methanol. Nevertheless, the investigated superheated water and methanol phase and their hydrogen bond dynamics may resemble that of a supercritical fluid, which is very difficult to study with other techniques. So, what is compared here between the two liquids is the hydrogen bond dynamics and the ability and tendency to form hydrogen bonds and clusters in such superheated phases on very short timescales. Within such a hypothesis the experimental results together with our molecular dynamics simulations are interpreted as follows. Superheated (and possibly supercritical temperature) water appears to consist mainly of monomers and relatively small high-temperature aggregates and clusters. Larger aggregates are built only on a time scale of several hundreds of picoseconds after expansion of the superheated phase - which is not relevant for supercritical reactions environments. Superheated (and possibly supercritical temperature) methanol, however, appears to consist of larger aggregates such as observed here on very short time scales. The aggregates in the laser experiment preserve the hydrogen bonds, which were present in the initial superheated liquid phase. The different dynamics of the two phases in the IR-pump and EUV-probe experiments could ultimately be explained with their varying interaction potentials on a molecular level. Two unequal interaction sites, as in the case of methanol, result in large aggregate formation, where the weaker interaction is broken. Water with two equal interaction sites, however, tends to built monomers increasing the entropy and reducing the free energy.

If we take into account the short time behavior of the superheated fluids (before significant expansion of the hot phase) and exclude the long time behavior, which may not be relevant for a comparison with true supercritical phases, we find two quite different features of methanol and water under high temperature conditions. Quite counter-intuitively, water appears to behave more like a hot monomer fluid under supercritical conditions, while methanol preserves hydrogen bonds - even under conditions much above its boiling temperature.

Most interestingly, these findings may explain why water is much more reactive in the supercritical phase than methanol. Although, here we study artificially heated 'model systems', a comparison of both phases in combination with theory enables us to obtain information about superheated phases which can hardly be obtained with other technologies for supercritical phases in thermodynamical equilibrium.

Finally, the varying dynamic properties between the two liquids may also alter energy transfer and transport processes and/or lower activation barriers in chemical reactions. These differences, in turn, can be used to improve or control chemical reactivity differently in these two different supercritical solvents.

\section{Acknowledgements}

The authors thank the Fonds der Chemischen Industrie, the Volkswagenstiftung, and the Deutsche Forschungsgemeinschaft DFG for financial support (Project AB63/12-1). E.V.M. also thanks Fondecyt No. 11121179 and Grant ICM No. 120082. F.W. and H.G. thank for funding by the DFG, SFB 755.

\section{References}

1 M. R. Manaa, Chemistry at Extreme Conditions, Elsevier Science, New York, 2005.

2 N. Akiya and P. E. Savage, Chem. Rev., 2002, 102, 2725.

3 P. E. Savage, Chem. Rev., 1999, 99, 603-621.

4 J. R. Errington and P. G. Debenedetti, Nature, 2001, 409, 318-321.

5 P. G. Debenedetti, Metastable Liquids: Concepts and Principles, Princeton Academic Press, Princeton, New Jersey, 1996.

6 O. Kajimoto, Chem. Rev., 1999, 99, 355.

7 R. von Benten, A. Charvat, O. Link, B. Abel and D. Schwarzer, Chem. Phys. Lett., 2004, 386, 325-329.

8 A. Charvat, B. Stasicki and B. Abel, J. Phys. Chem. A, 2006, 110, 3297-3306.

9 H. H. Hamann, A. Charvat, B. Abel, S. A. Kovalenko and A. A. Kachanov, J. Chem. Phys., 1997, 106, 3103-3116.

10 F. D. Mango, J. W. Hightower and A. T. James, Nature, 1994, 368, 536.

11 E. L. Shock, Nature, 1994, 368, 499.

12 J. S. Seewald, Nature, 1994, 370, 285.

13 L. C. Price, Nature, 1994, 370, 253.

14 F. N. Spiess, K. C. Macdonald, T. Atwater, R. Ballard, A. Carranza, D. Cordoba, C. Cox, V. M. Diaz-Garcia, J. Francheteau, J. Guerrero, J. Hawkins, J. Haymon, R. Hessler, T. Juteau, M. Kastner, R. Larson, B. Luyendyk, J. D. Macdougall, S. Miller, W. Normark, J. Orcult and C. Rangin, Science, 1980, 1421.

15 W. J. Jones, J. A. Leigh, F. Mayer, C. R. Woese and R. S. Wolfe, Arch. Microbiol., 1983, 136, 254.

16 C. J. Bult, O. White, G. J. Olsen, L. Zhou, R. D. Fleischmann, C. J. Sutton, J. A. Blake, L. M. Fitzgerald, R. A. Clayton, J. D. Gocayne, A. R. KErlavage, B. A. Doughery, J. F. Tomb, M. D. Adams, C. I. Reich, R. Overbeck, E. F. Kirkness, K. G. Weinstock, J. M. Merrick, A. Glodek, J. L. Scott, 
N. S. M. Geohagen, J. F. Weidmann, J. L. Fuhrmann, D. Nguyen, T. R. Utterback, J. M. Kelley, J. D. Perterson, P. W. Sadow, M. C. Hanna, M. D. Cotton, K. M. Roberts, M. A. Hurst, B. P. Kaine, M. Borodovsky, H. P. Klenk, C. M. Fraser, H. O. Smith, C. R. Woese and J. C. Venter, Science, 1996, 273, 1058.

17 J. Yao and R. F. Evilia, J. Am. Chem. Soc., 1994, 116, 11229.

18 P. Wernet, D. Testemale, J.-L. Hazemann, R. Argoud, P. Glatzel, L. G. M. Petterson, A. Nilsson and U. Bergmann, J. Chem. Phys., 2005, 123, 154503.

19 P. Postorino, R. H. Tromp, M. A. Ricci, A. K. Spoper and G. W. Neilson, Nature, 1993, 366, 668.

20 R. H. Tromp, P. Postorino, G. W. Neilson, M. A. Rici and A. K. Soper, J. Chem. Phys., 1994, 101, 6210.

21 J. E. Enderby and G. W. Neilson, in Water: A Comprehensive Treatise, ed. F. Franks, Plenum Press, New York, 1979, vol. 6.

22 A. K. Soper and M. G. Phillips, Chem. Phys. Lett., 1986, $107,47$.

23 A. K. Soper, F. Bruni and M. A. Ricci, J. Chem. Phys., 1997, 106, 247.

24 A. G. Kalinichev and J. D. Bass, Chem. Phys. Lett., 1994, 231, 301.

25 A. A. Chialvo and P. T. Cummings, J. Chem. Phys., 1994, 101, 4466.

26 Y. E. Gorbaty and Y. N. Demianets, Chem. Phys. Lett., 1983, 100, 450.

27 K. Yamanaka, T. Yamaguchi and H. Wakita, J. Chem. Phys., 1994, 101, 9830.

28 Y. E. Gorbaty and A. G. LKalinichev, J. Phys. Chem., 1995, 99, 5336.

29 E. U. Franck and K. Roth, Discuss. Faraday Soc., 1967, 1967, 108.

30 W. Kohl, H. A. Lindner and E. U. Franck, Ber. Bunsen-Ges., 1991, 95, 1586.

31 C. I. Ratcliffe and D. E. Irish, J. Phys. Chem., 1982, 86, 4897.

32 G. E. Walrafen, Y. C. Chu and G. J. Piermarini, J. Phys. Chem., 1996, 100, 10363.

33 R. D. Mountain, J. Chem. Phys., 1989, 90, 1866.

34 E. S. Fois, M. Sprik and M. Parrinello, Chem. Phys. Lett., 1994, 223, 411.

35 T. I. Mizan, P. E. Savage and R. M. Ziff, J. Phys. Chem., 1996, 100, 403.

36 T. Ebukuro, A. Takami, Y. Oshima and S. Koda, J. Supercrit. Fluids, 1999, 15, 73.

37 N. Asahi and Y. Nakamura, Chem. Phys. Lett., 1998, 290, 63. 38 N. Asahi and Y. Nakamura, J. Chem. Phys., 1998, 109, 9879.

39 M. M. Hoffmann and M. S. Conradi, J. Phys. Chem. B, 1998, 102, 263.

40 S. Bai and C. R. Yonker, J. Phys. Chem. A, 1998, 102, 8641. 41 M. Chalaris and J. Samios, J. Phys. Chem. B, 1999, 103, 1161. 42 M. Chalaris and J. Samios, Pure Appl. Chem., 2004, 76, 203. 43 T. Yamaguchi, C. J. Benmore and A. K. Soper, J. Chem. Phys., 2000, 112, 8976.

44 T. Yamaguchi, K. Yoshida, M. Shimohira and M. Nagao, Int. Symp. Molecular Thermodynamics and Molecular Simulations (MTM '03), Sendai, Japan, 2003.
45 M. L. Cowan, B. D. Bruner, N. Huse, J. R. Dwyer, B. Chugh, E. T. Nibbering, T. Elsaesser and R. J. Miller, Nature, 2005, 434, 199-202.

46 D. Schwarzer, J. Lindner and P. Vöhringer, J. Chem. Phys., 2005, 123, 161105.

47 O. Link, E. Lugovoy, K. Siefermann, Y. Liu, M. Faubel and B. Abel, Appl. Phys. A: Mater. Sci. Process., 2009, 96, 117-135. 48 O. Link, E. Vöhringer-Martinez, E. Lugovoj, X. Liu, K. Siefermann, M. Faubel, H. Grubmüller, R. B. Gerber, Y. Miller and B. Abel, Faraday Discuss., 2009, 141, 67-79.

49 B. Abel, J. Assmann, M. Buback, M. Kling, S. Schmatz and J. Schroeder, Angew. Chem., Int. Ed., 2003, 42, 299-303.

50 K. Siegbahn, D. Hammond, H. Fellner-Feldegg and E. F. Barnett, Science, 1972, 176, 245-252.

51 B. Winter and M. Faubel, Chem. Rev., 2006, 106, 1176-1211. 52 M. Faubel and T. Kisters, Nature, 1989, 339, 527-529.

53 M. Faubel, S. Schlemmer and J. P. Toennies, Z. Phys. D: At., Mol. Clusters, 1988, 10, 269.

54 X. F. Li, A. L'Huillier, M. Ferray, L. A. Lompré and G. Mainfray, Phys. Rev. A, 1989, 39, 5751.

55 K. R. Siefermann, Y. Liu, E. Lugovoy, O. Link, M. Faubel, U. Buck, B. Winter and B. Abel, Nat. Chem., 2010, 2, 274-279.

56 M. Faubel, K. R. Siefermann, Y. Liu and B. Abel, Acc. Chem. Res., 2012, 45, 120-130.

57 M. Michaud, A. Wen and L. Sanche, Radiat. Res., 2003, $159,3$.

58 N. Ottosson, M. Faubel, S. E. Bradforth, P. Jungwirth and B. Winter, J. Electron. Spectrosc. Relat. Phenom., 2010, 177, 60. 59 D. Emfietzoglou and H. Nikjoo, Radiat. Res., 2005, 163, 98. 60 D. Emfietzoglou, I. Kyriakou, I. Abril, R. Garcia-Molina, I. D. Petsalakis, H. Nikjoo and A. Pathak, Nucl. Instrum. Methods Phys. Res., Sect. B, 2009, 267, 45-52.

61 S. Thürmer, R. Seidel, M. Faubel, W. Eberhardt, J. C. Hemminger, S. E. Bradforth and B. Winter, Phys. Rev. Lett., 2013, 111, 173005.

62 D. van der Spoel, E. Lindal, B. Hess, G. Groenhof, A. E. Mark and H. J. C. Berendsen, J. Comput. Chem., 2005, 26, 1701-1708.

63 H. J. C. Berendsen, J. P. M. Postma, A. DiNicola and J. R. Haak, J. Chem. Phys., 1984, 81, 3684-3690.

64 H. J. C. Berendsen, D. van der Spoel and R. van Drunen, Comput. Phys. Commun., 1995, 91, 43-56.

65 W. L. Jorgensen, J. Chandrasekar, J. D. Madura, R. W. Impey and M. Klein, J. Chem. Phys., 1983, 79, 926-935.

66 G. Lamoureux, A. D. MacKerell and B. Roux, J. Chem. Phys., 2003, 119, 5185.

67 F. Franks, Water-a comprehensive treatise-vol. 1: the physics and physical chemistry of water, Plenum Press, 1972.

68 O. Kajimoto, Chem. Rev., 1999, 99, 355-389.

69 M. Faubel, in Adv. Series in Physical Chemistry, ed. C. Y. Ng, World Scientific, New York, 2000, vol. 10A.

70 S. Barth, M. Oncak, V. Ulrich, M. Mucke, T. Lischke, P. Slavícek and U. Hergenhahn, J. Phys. Chem. A, 2009, 113, 13519-13527.

71 O. Björneholm, F. Federmann, S. Kakar and T. Moller, J. Chem. Phys., 1999, 111, 546-550. 
72 G. Öhrwall, R. F. Fink, M. Tchaplyguine, L. Ojamaë, M. Lundwall, R. R. T. Marinho, A. N. de Brito, S. L. Sorensen, M. Gisselbrecht, R. Feifel, T. Rander, A. Lindblad, J. Schulz, L. J. Saethre, N. Martensson, S. Svensson and O. Björneholm, J. Chem. Phys., 2005, 123, 054310.

73 U. Emmerichs, S. Woutersen and H. J. Bakker, J. Opt. Soc. Am., 1997, 14, 1480.

74 M. F. Kropman and H. J. Bakker, Science, 2001, 291, 2118-2120.
75 M. Schmeisser, H. Iglev and A. Laubereau, J. Phys. Chem. B, 2007, 111, 11271-11275.

76 M. Faubel, B. Steiner and J. P. Toennies, J. Chem. Phys., 1997, 106, 9013-9031.

77 I. Skarmoutsos and J. Samios, J. Phys. Chem. B, 2006, 110, 21931-21937.

78 E. Espinosa, E. Molins and C. Lecomte, Chem. Phys. Lett., 1998, 285, 170-173.

79 C. R. Brundle and D. W. Turner, Proc. Roy. Soc. A, 1968, 307, 27. 